\title{
Mapas de España de corrosividad del zinc en atmósferas rurales ${ }^{\left({ }^{\circ}\right.}$
}

\begin{abstract}
Resumen
El interés en la elaboración de mapas de corrosividad atmosférica radica en su utilidad para disponer de información sobre el alcance del fenómeno de corrosión atmosférica en un determinado punto geográfico. Con ello se facilitaría la selección del material metálico más adecuado, tanto desde el punto de vista de resistencia a la corrosión como económicamente, y definir, asimismo, el tipo de protección idónea para una durabilidad determinada. En la práctica se trata de una tarea difícil, debido a los numerosos factores climáticos de los que depende la corrosión atmosférica. Este trabajo resume un proyecto financiado por BP Solar para el desarrollo de mapas de España para la corrosividad atmosférica del zinc, tanto de corrosión anual como de corrosión a largo plazo (15 años), que determinará el tipo de protección requerido en las estructuras metálicas utilizadas en sistemas fotovoltaicos para atmósferas rurales, prácticamente exentas de contaminación. La metodología seguida ha sido la aplicación de ecuaciones dosis/respuesta (funciones de daño), a partir de las cuales se estima la velocidad de corrosión en función de variables meteorológicas. Estas variables se han obtenido a partir de información procedente de estaciones meteorológicas ubicadas en la España peninsular.
\end{abstract}

B. Chico*, D. de la Fuente*, J.M. Vega* y M. Morcillo*

\section{Corrosivity maps of Spain for zinc in rural atmospheres}

\begin{abstract}
Atmospheric corrosivity maps provide useful information on the extent of atmospheric corrosion phenomena in a given geographic scope. The preparation of such maps helps designers to select the most suitable metallic material in terms of corrosion resistance and economy and to define the right type of protection for a given durability. This is a difficult task, due to the numerous climate-related factors upon which atmospheric corrosion depends. This work summarizes a funded project by BP Solar to develop atmospheric corrosivity maps of Spain for zinc considering both annual corrosion and long term ( 15 years) corrosion, which will determine the protection required for the metal structures used in photovoltaic systems, for practically pollution-free rural atmospheres. The method used has been to apply dose/response equations (damage functions) to estimate the corrosion rate as a function of meteorological variables. These variables have been obtained from information of meteorological stations placed in mainland Spain.
\end{abstract}

Keywords Corrosion map; Rural atmosphere; Zinc; Climate; Damage function; Mainland Spain.

\section{INTRODUCCIÓN}

Los mapas de corrosividad atmosférica son de gran utilidad ya que, además de representar visualmente el dato de corrosión, se pueden emplear como una herramienta para poder disponer de información acerca del alcance del fenómeno de corrosión atmosférica en un determinado punto geográfico y seleccionar, de esta manera, el material metálico más adecuado, tanto desde el punto de vista de resistencia a la corrosión como del económico, y definir, asimismo, el tipo de protección anticorrosiva o bien, poder estimar el espesor de un determinado recubrimiento anticorrosivo para una durabilidad determinada, así como la frecuencia en las operaciones de mantenimiento.

Evidentemente, la elaboración de estos mapas no es tarea fácil, habida cuenta de que el fenómeno de la corrosión atmosférica depende de un gran número de factores climáticos, tanto meteorológicos como de contaminación ${ }^{[1]}$. A este elevado número de variables, habría que sumar, asimismo, su variación con el tiempo, así como las posibles interrelaciones entre ellas. Si a todo ello se une que, además, se

\footnotetext{
(•) Trabajo recibido el día 1 de Junio de 2010 y aceptado en su forma final el día 1 de Septiembre de 2010.

* Departamento de Ingeniería de Superficies, Corrosión y Durabilidad, Centro Nacional de Investigaciones Metalúrgicas (CENIM, CSIC), Avda. Gregorio del Amo, 8. 28040-Madrid.
} 
puedan interpretar de manera sencilla, hace que la elaboración sea aún más compleja.

Existen diferentes enfoques para la construcción de estos mapas ${ }^{[2]}$. Uno de ellos sería mediante la obtención directamente del dato de corrosividad atmosférica, mediante su efecto sobre distintos metales de referencia (acero al carbono, zinc, cobre y aluminio) expuestos en distintos lugares geográficos. Evidentemente, ello supondría el disponer de una amplia red de estaciones de ensayo y cubrir, prácticamente, el área que se quiere estudiar. Por ello, si la pretensión es elaborar el mapa de corrosividad atmosférica de un país, se tendrían que disponer de miles de estaciones, con el elevado coste que ello podría suponer, y aún así, las estimaciones podrían ser muy arriesgadas.

Otro método resulta de estimar el dato de corrosión en una determinada región a partir de datos ambientales disponibles y de su efecto sobre distintos materiales mediante la experimentación a nivel de laboratorio.

Una tercera metodología seguida en la práctica es a partir del conocimiento de funciones reales dosis/respuesta existentes en una determinada región geográfica. También se las denomina funciones de daño, y son ecuaciones obtenidas mediante el tratamiento estadístico de los datos, y que permiten predecir la velocidad de corrosión anual a partir de parámetros meteorológicos y de contaminación. Es posible encontrar en la literatura especializada ${ }^{[3}$ y 4$]$ este tipo de funciones para un gran número de metales, especialmente acero y zinc, y son ecuaciones que incorporan frecuentemente como variables independientes, la concentración de $\mathrm{SO}_{2}$, la concentración de cloruros (salinidad atmosférica) en zonas cercanas al mar, y parámetros representativos de la humectación de la superficie metálica (días de lluvia al año, humedad relativa, tiempo de humectación de la superficie metálica, etc.).

Los datos se podrían ajustar a la siguiente ecuación lineal;

$$
C=a_{1}+a_{2} H R+a_{3} P+a_{4} T+a_{5} T D H+a_{6} S+a_{7} C l
$$

donde los coeficientes $\mathrm{a}_{1}$ - $\mathrm{a}_{7}$ son constantes; $\mathrm{C}$, la corrosión anual, en $\mu \mathrm{m} ; \mathrm{HR}$, la humedad relativa media anual, en \%; P, los días de precipitación al año; T, la temperatura media anual, en ${ }^{\circ} \mathrm{C}$; TDH (tiempo de humectación), fracción anual del número de horas/año en que la $\mathrm{HR}>80 \%$ y $\mathrm{T}>0{ }^{\circ} \mathrm{C}$; $\mathrm{S}$, la contaminación por $\mathrm{SO}_{2}$ en $\mathrm{mg} / \mathrm{m}^{2}$. día y $\mathrm{Cl}$, la contaminación por cloruros en $\mathrm{mg} / \mathrm{m}^{2}$.día.

Si se anulan los términos correspondientes al efecto de los contaminantes, la ecuación de regresión da información sobre la corrosión en atmósferas puras en función de las variables meteorológicas. Con los mapas elaborados a partir de estos criterios se pueden hacer previsiones sobre el comportamiento en atmósferas rurales prácticamente exentas de contaminación, que comprenden, por lo normal, la mayor parte del territorio de un país. Asimismo, la corrosión en atmósferas rurales adquiere una gran importancia práctica teniendo en cuenta la profusión de equipos (maquinaria diversa, redes de distribución de energía eléctrica, instalaciones agrícolas, etc.) que durante su vida en servicio están expuestos a este tipo de atmósferas.

De acuerdo a la normativa ISO $9223^{[5]}$, se considera atmósfera rural (atmósfera clasificada como SOPO) aquella que tiene un nivel de cloruros $\leq 3$ $\mathrm{mg} / \mathrm{m}^{2}$.d y una contaminación por $\mathrm{SO}_{2} \leq 10 \mathrm{mg} / \mathrm{m}^{2}$.d.

Otro dato de suma importancia, lo constituye la corrosión a largo plazo. Desgraciadamente, en la bibliografía escasean datos de corrosión atmosférica de metales para períodos de exposición prolongados (10-20 años o más ${ }^{[6]}$, a pesar de que el conocimiento de este dato es de enorme interés a la hora de dictaminar las medidas de protección más adecuadas. Existen dos métodos para realizar estimaciones de corrosión a largo plazo. Un método consiste en la aplicación de la Norma ISO $9224^{[7]}$ donde se han establecido unos valores guía de corrosión a partir de estudios de corrosión atmosférica de larga duración llevados a cabo en diferentes países.

Un segundo método sería mediante la aplicación de una función potencial (ley bilogarítmica). Es conocido que el proceso de corrosión atmosférica a largo plazo suele ser asimilable con buena aproximación a funciones del tipo;

$$
C=A \cdot t^{n}
$$

donde C es la corrosión al cabo de $\mathrm{t}$ años y $\mathrm{A}$ es la corrosión durante el primer año de exposición. El valor de $\mathrm{n}$ depende del metal, del tipo de atmósfera y de las condiciones de exposición. Si $n=0,5$; tendríamos el caso de un proceso de corrosión atmosférica ideal controlado por difusión, cuando todos los productos de corrosión permanecen sobre la superficie como capa no perturbada. Si el coeficiente de difusión decrece con el tiempo, por ejemplo, debido a un proceso de compactación de la herrumbre o decrecimiento de la porosidad de la capa de productos de corrosión, $n$ adquiere valores por debajo de 0,5. Por el contrario, si el proceso de difusión se acelera, por ejemplo, por disolución, desprendimiento, etc., de la capa de productos de corrosión, $n$ adquiere valores por encima de 0,5 hasta un valor límite de 1 . Por ello, se considera a $n$ como un indicador del comportamiento físico-químico de la capa de productos de corrosión. 
El objetivo del presente trabajo ha sido la elaboración de mapas de velocidad de corrosión del zinc de la España peninsular, tanto de corrosión anual como de corrosión a largo plazo (15 años), comprendiendo únicamente las atmósferas rurales. La velocidad de corrosión se estima a partir de una ecuación dosis/respuesta, seleccionada de estudios de campo realizados ${ }^{[2-4]}$.

\section{METODOLOGÍA}

\subsection{Análisis de las funciones de daño para estimar la velocidad de corrosión}

Se ha realizado una búsqueda en la bibliografía de funciones de daño a partir de las cuales se pudiese estimar la velocidad de corrosión anual del zinc en atmósferas rurales en función de diversos parámetros meteorológicos ${ }^{[2,6 \text { y } 8]}$.

Un estudio llevado a cabo por M. Morcillo y S. Feliu $^{[2]}$, iniciado en España en el año 1976, se encaminó hacia la obtención de datos de corrosión en atmósferas libres de contaminación. Se pretendía, de esta manera, poder evaluar el efecto exclusivo de las variables meteorológicas sobre la corrosión metálica. Se seleccionaron tres zonas de España, meteorológicamente diferenciadas: Centro, Noroeste y Sur, ubicando estaciones de ensayo alejadas de todo núcleo urbano o industrial y de la costa. En total se ubicaron unas 100 estaciones de ensayo.

Dentro de este estudio, y con el fin de determinar estadísticamente la influencia de las variables meteorológicas en la corrosión atmosférica, se acudió a la técnica de regresión lineal múltiple. Una de las funciones de corrosión que se consideró fue la siguiente;

$$
C=f_{1}(H R, T, L)
$$

donde C es la corrosión anual del metal (expresada en $\mu \mathrm{m}), \mathrm{HR}$ es la humedad relativa media anual (expresada en \%), T es la temperatura media anual (expresada en ${ }^{\circ} \mathrm{C}$ ) y $\mathrm{L}$ el número de días de lluvia al año.

La función resultante para el caso del zinc y su coeficiente de correlación (R) correspondiente fue;

$$
C=-0,00603 H R+0,00380 T+0,00973 L+0,597 R=0,705
$$

El relativamente alto coeficiente de correlación obtenido para este metal indica una influencia significativa de la meteorología en la corrosión atmosférica del zinc. Asimismo, también se deduce que la variable $L$ (días de lluvia) presenta mayor influencia que los otros dos parámetros ( $T$ y HR).

Por tanto, se ha considerado conveniente seleccionar esta función de daño para poder estimar la corrosión anual del zinc a partir de variables climatológicas, metodología que se describe en los apartados siguientes.

\subsection{Obtención de los datos meteorológicos}

Para la obtención de los datos meteorológicos se ha acudido a la información publicada en el "Atlas Nacional de España”. Climatología $2^{\underline{a}}$ Edición ${ }^{[9]}$. En este Atlas están publicados los Mapas de España de los diferentes elementos que caracterizan el clima de una región. Se realiza una descripción del estado climático para un período de treinta años, comprendiendo el intervalo 1971-2000. No se trata de un período estándar, pero permite dar una visión actual del clima en España.

Dentro de este capítulo del Atlas se describe la metodología seguida para la obtención de los mapas climáticos. Estos se elaboraron a partir de información procedente de un total de 143 estaciones principales, 1.504 estaciones termométricas y 4.835 pluviométricas ${ }^{[9]}$. Las estaciones principales efectúan medidas instrumentales continuas de las diferentes variables meteorológicas (temperatura, precipitación, presión, humedad, viento, horas de sol y radiación), mientras que en las estaciones termométricas se registra la temperatura y precipitación, y en las pluviométricas únicamente ésta última variable.

Estos mapas climáticos editados en el Atlas se han trazado con el sistema de información geográfica de la Agencia Estatal de Meteorología (AEMET). Así, para la interpolación de las variables climáticas, se empleó el método de Kriging. Dicho método provee, a partir de una muestra de puntos, ya estén regular o irregularmente distribuidos, valores estimados de aquellos sitios donde no hay información, sin sesgo y con una varianza mínima conocida.

En el caso de la lluvia, y dada la gran variabilidad espacial de este parámetro, para su adecuada descripción es necesario un elevado número de datos. Debido a la irregular distribución espacial de la precipitación en España, dividieron el territorio en zonas más pequeñas y climáticamente homogéneas, que coincidían básicamente con las principales cuencas hidrográficas. Dentro de cada una de estas zonas aplicaron un modelo de regresión diferente para cada mes del año. 


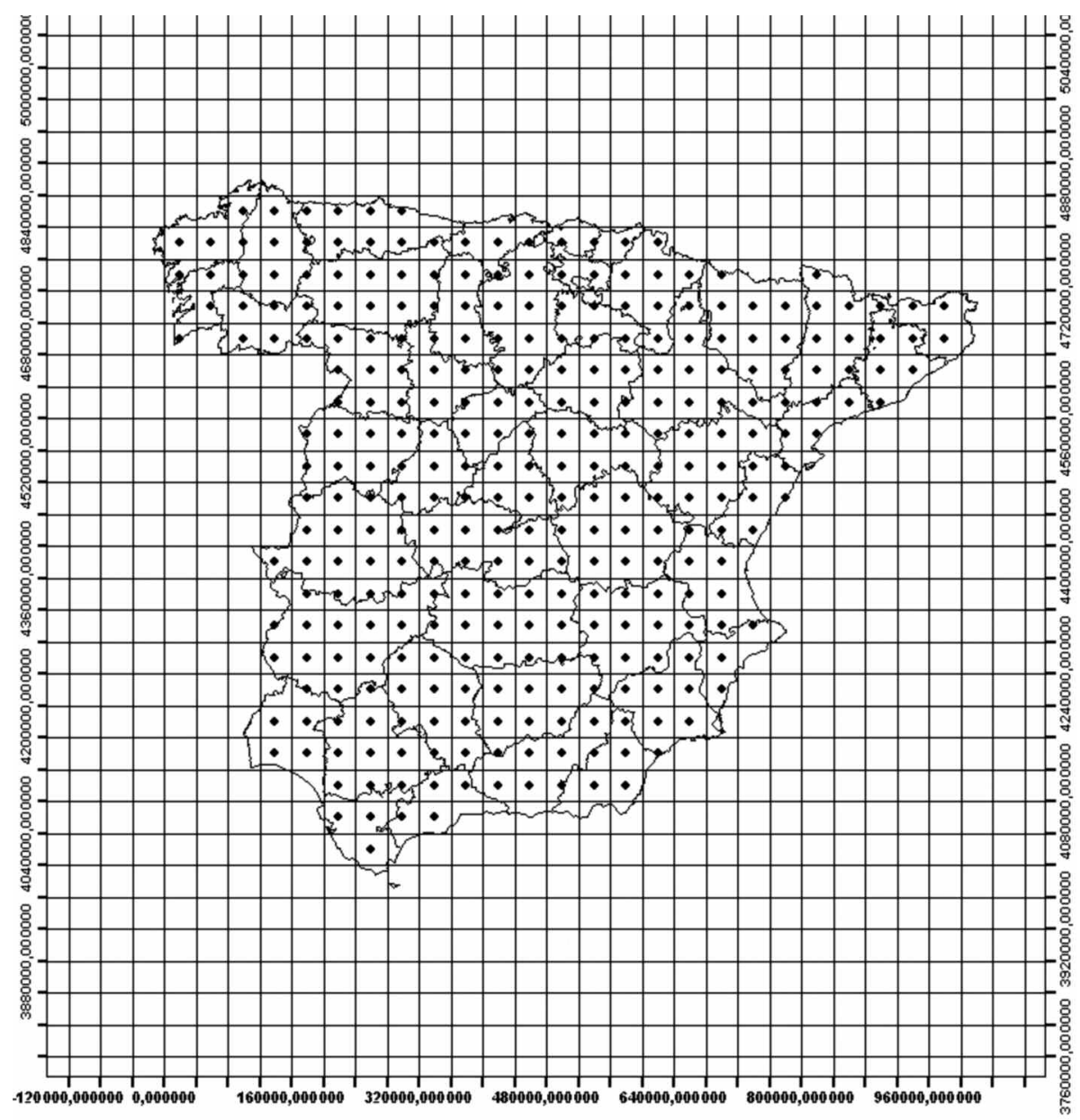

Figura 1. Gradilla geo-referenciada utilizada sobre los mapas climáticos editados en el Atlas Nacional de España ${ }^{[9]}$, para la obtención de los datos climatológicos.

Figure 1. Geo-referenced gradient used on climate maps published in the National Atlas of Spain ${ }^{[9]}$ for the obtainment of climate data.

Para poder extraer de los mapas climáticos ${ }^{[9]}$ la información de las variables climatológicas en diferentes puntos geográficos, y así aplicar la ecuación (4) y estimar el dato de velocidad de corrosión del zinc, se dibujó sobre cada uno de ellos una gradilla correspondiente a un mapa de España geo-referenciado, tal y como se muestra en la figura 1 . Dentro de cada una de estas casillas se tomó el valor medio del parámetro climatológico en cuestión, dándole unas coordenadas XY correspondientes al punto central de cada casilla de la gradilla. En total se tomaron datos de un total de 306 puntos distribuidos homogéneamente por toda España.

Por otro lado, y con el objetivo tanto de contrastar la información meteorológica obtenida de los mapas, como la de manejar datos climatológicos más recientes, se solicitaron al AEMET datos de humedad relativa, temperatura y días de lluvia para un período de los últimos 15 años: 1994-2008. En este caso, la información recopilada procedía de 223 estaciones con datos de humedad relativa, 346 estaciones con datos de temperatura y 118 estaciones con datos de días de lluvia. La distribución de estas estaciones se muestra en la figura 2 .

\section{ELABORACIÓN DE LOS MAPAS DE CORROSIÓN ATMOSFÉRICA DEL ZINC}

\subsection{Mapas de corrosión anual}

Mediante la sustitución de los datos climatológicos en la ecuación (4), ha sido posible estimar una velocidad anual de corrosión del zinc para cada uno de los 


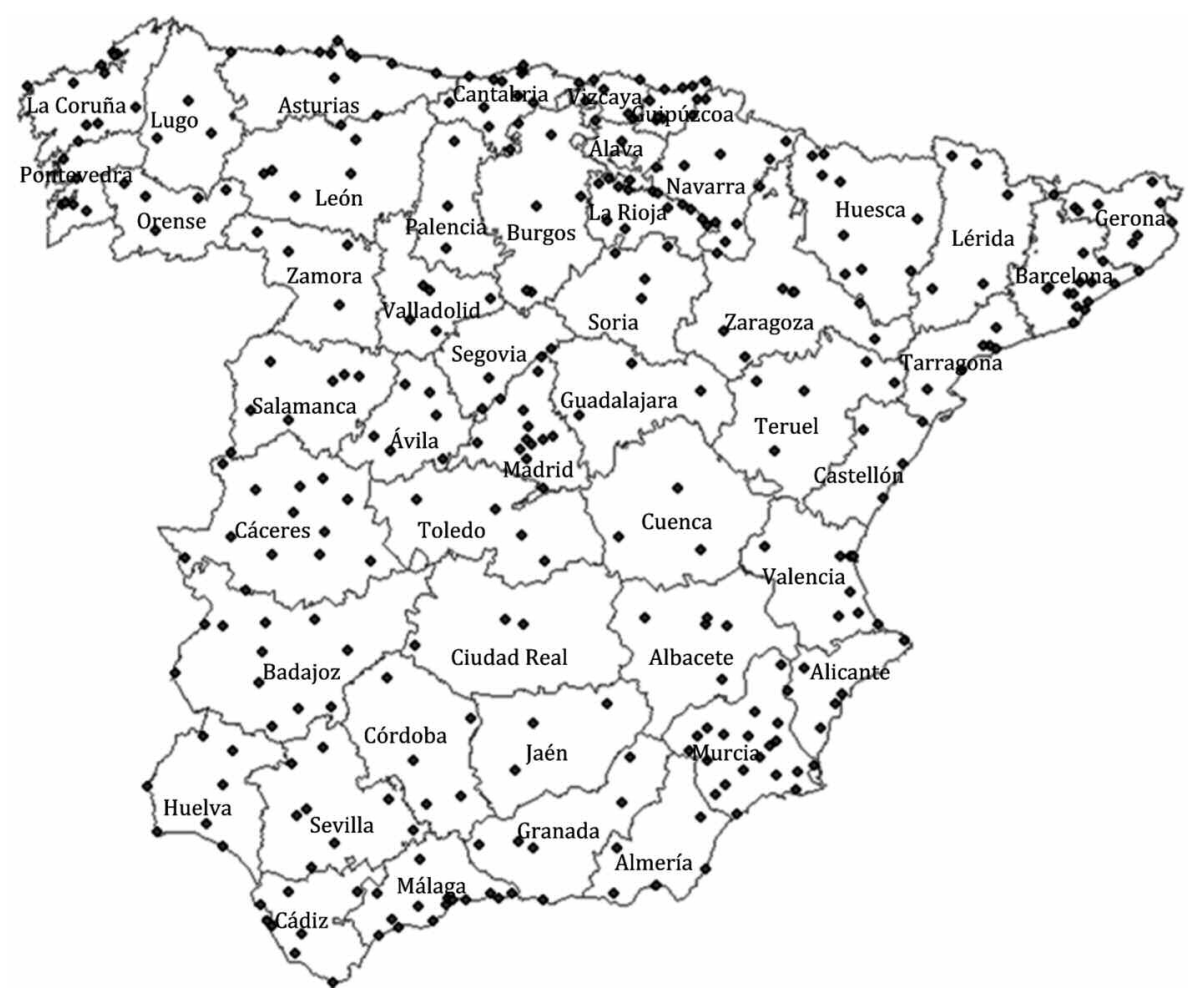

Figura 2. Red de estaciones con información meteorológica suministrada por AEMET, para el período 1994-2008.

Figure 2. AEMET meteorological information stations network for the period 1994-2008.

puntos geográficos que se observan en la figura 1. Posteriormente, para la elaboración del mapa de España de corrosión del zinc, se ha empleado el software ArcGis 9.1 con la extensión de "Análisis Espacial". Asimismo, se ha utilizado el método de interpolación de Kriging para la representación de los datos con un tamaño de celda de $500 \mathrm{~m}$ x $500 \mathrm{~m}$.

El mapa elaborado se representa en la figura 3. La velocidad de corrosión anual del zinc se ha clasificado en 4 intervalos, llegándose, mediante el método de interpolación empleado, a un máximo de velocidad de corrosión de 1,61 $\mu \mathrm{m} /$ año.

Se observa la existencia de un primer intervalo de corrosión $\leq$ 0,7 $\mu \mathrm{m} /$ año (el valor mínimo de corrosión que se establece mediante el método de interpolación empleado es 0,62 $\mu \mathrm{m} /$ año), que correspondería prácticamente con el límite superior de la categoría C2 establecida por ISO 9223[5] (Tabla I). Esta zona de corrosión inferior correspondería prácticamente a las provincias de Almería y Murcia, como se puede observar en el mapa.

En los mapas obtenidos, el intervalo de velocidad que establece la norma ISO para $\mathrm{C} 3$, se ha dividido a su vez en tres intervalos: (a) $0,7<\mathrm{v}_{\mathrm{Zn}} \leq 1,0 \mu \mathrm{m} / \mathrm{año}$;
Tabla I. Intervalos de corrosión del zinc para las diferentes categorías de corrosividad[5]

Table I. Zinc corrosion intervals for different corrosivity categories ${ }^{[5]}$

\begin{tabular}{lc}
\hline $\begin{array}{l}\text { Categoría de } \\
\text { corrosividad }\end{array}$ & $\begin{array}{c}\text { Velocidad de corrosión } \\
\text { del zinc, } \mu \text { m/año }\end{array}$ \\
\hline C1 (muy baja) & $\mathrm{v}_{\mathrm{Zn}} \leq 0,1$ \\
C2 (baja) & $0,1<\mathrm{v}_{\mathrm{Zn}} \leq 0,7$ \\
C3 (media) & $0,7<\mathrm{v}_{\mathrm{Zn}} \leq 2,1$ \\
C4 (alta) & $2,1<\mathrm{v}_{\mathrm{Zn}} \leq 4,2$ \\
C5 (muy alta) & $4,2<\mathrm{v}_{\mathrm{Zn}} \leq 8,4$ \\
\hline
\end{tabular}

(b) $1,0<\mathrm{v}_{\mathrm{zn}} \leq 1,3 \mu \mathrm{m} / \mathrm{año}$, (c) $1,3<\mathrm{v}_{\mathrm{zn}}$ $\leq 1,7 \mu \mathrm{m} /$ año para las distintas regiones de España.

Es importante comparar estas estimaciones con datos reales de corrosión anual obtenidos en España. Para ello, en la tabla II se exponen datos de campo de velocidad de corrosión del zinc obtenidos en atmósferas rurales de España ${ }^{[2]}$. Son datos de corrosión que corresponden a dos períodos anuales distintos. 


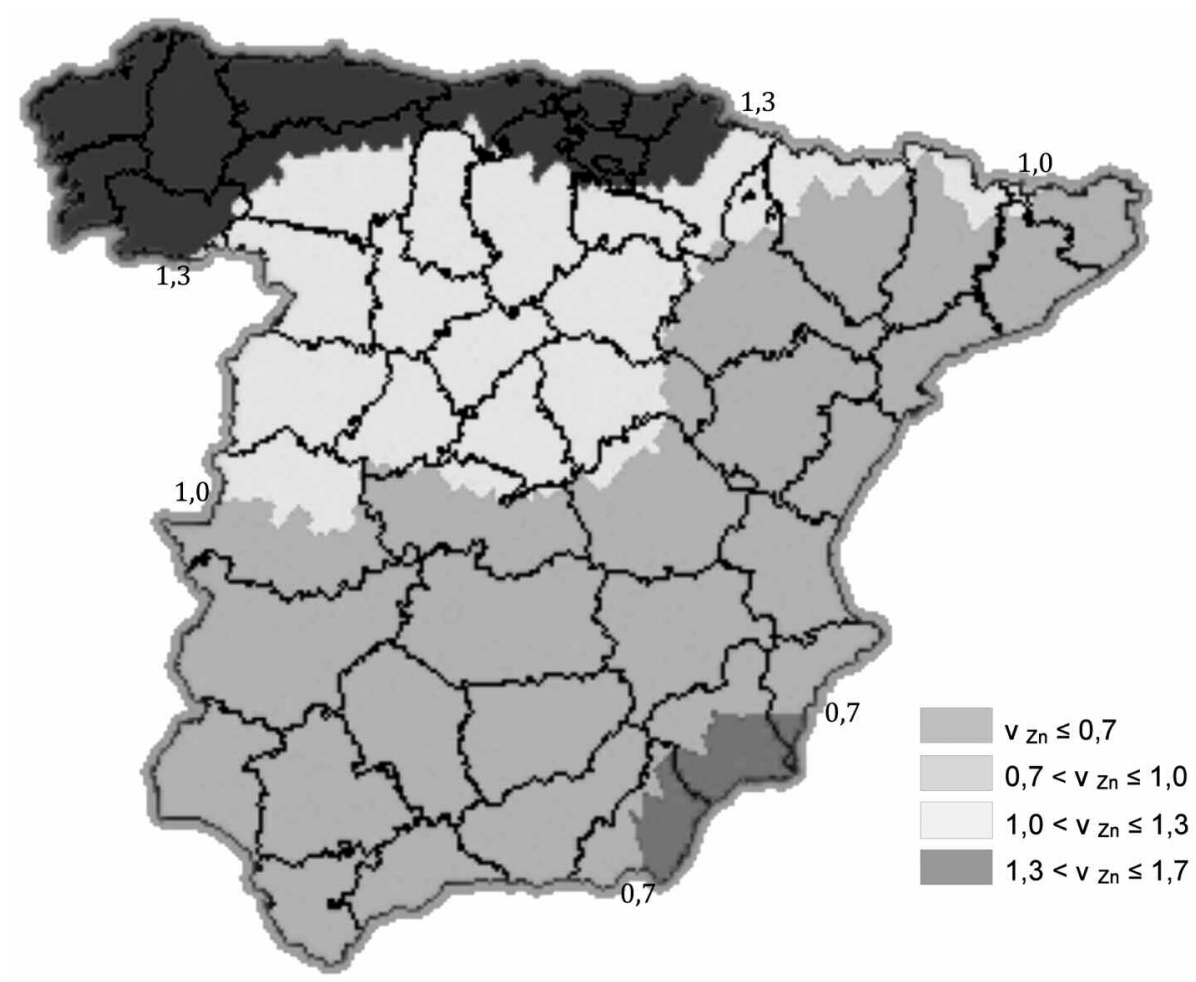

Figura 3. Mapa de España de velocidad de corrosión anual de zinc $\left(\mathrm{V}_{\mathrm{Zn}}\right.$ en $\mu \mathrm{m} / \mathrm{año}$ ).

Figure 3. Map of Spain for annual zinc corrosion rate $\left(V_{Z n}\right.$ in $\left.\mu m / y e a r\right)$.

Tabla II. Datos de campo de velocidad de corrosión del zinc en atmósferas rurales

\section{Table II. Zinc corrosion rate field data in rural atmospheres}

\begin{tabular}{lccc}
\hline $\begin{array}{c}\text { Zona de } \\
\text { España }\end{array}$ & \multicolumn{3}{c}{ V Zinc $(\mu$ m/año) } \\
Grupo* & 1 $^{\text {er }}$ período & $\mathbf{2}^{\mathbf{0}}$ período \\
\hline Sur: & 1 & 0,78 & 0,91 \\
Málaga, & 2 & 0,61 & 0,56 \\
Granada, & 3 & 0,47 & 0,47 \\
Almería & 4 & 0,52 & 0,57 \\
\hline Noroeste: & & & \\
La Coruña, & 1 & 2,05 & 1,10 \\
Lugo, & 2 & 2,00 & 1,45 \\
Orense y & 3 & 1,30 & 0,79 \\
Pontevedra & 4 & 1,53 & 0,83 \\
\hline Centro: & & & \\
\hline Madrid, & 1 & 1,90 & 1,10 \\
Toledo, & 2 & 1,30 & 1,30 \\
Guadalajara, & 3 & 1,50 & 1,20 \\
Cáceres & 4 & 1,30 & 0,70 \\
\hline
\end{tabular}

* Grupos establecidos de estaciones de corrosión en función de la existencia de estaciones meteorológicas próximas a aquellas.
Comparando estos datos reales de velocidad de corrosión con las estimaciones que se obtienen mediante los mapas, se observa que no existen grandes desviaciones. Evidentemente, los datos mostrados en la tabla corresponden únicamente a dos años determinados, mientras que en los mapas la estimación se realiza a partir de un período más amplio de tiempo.

Como se ha indicado anteriormente, la velocidad de corrosión anual del zinc ha sido estimada a partir de la ecuación (4), la cual a su vez fue obtenida para atmósferas rurales. Por ello, no se deben aplicar estos mapas en zonas cercanas al mar, o bien en zonas industriales. En estas zonas la velocidad de corrosión del zinc es mayor debido a la influencia de la presencia de cloruros o bien de $\mathrm{SO}_{2}$ en la corrosión de este metal ${ }^{[10}$ y 11$]$.

Los resultados obtenidos empleando la misma función de daño, pero con los datos climatológicos correspondientes a los 15 últimos años suministrados por AEMET (Fig. 2), son muy similares. Los valores más bajos de velocidad de corrosión anual del zinc se obtienen en las zonas de Almería y Murcia, mientras que los más altos, corresponden nuevamente a la zona norte de España, fundamentalmente Galicia, Cantabria y País Vasco. 
MAPAS DE ESPAÑA DE CORROSIVIDAD DEL ZINC EN ATMÓSFERAS RURALES CORROSIVITY MAPS OF SPAIN FOR ZINC IN RURAL ATMOSPHERES

\subsection{Mapas de corrosión a largo plazo}

Los mapas de corrosión del zinc a largo plazo se han elaborado de acuerdo a un estudio llevado a cabo por Morcillo et al. ${ }^{[6]}$. Es un estudio que reporta información de datos experimentales de velocidad de corrosión atmosférica del zinc para un tiempo de exposición de 13-16 años, obtenidos en diferentes atmósferas de España, así como de datos de larga duración obtenidos en otras partes del mundo.

Para el caso del zinc, todos los datos de corrosión se ajustan razonablemente bien a una ecuación de tipo $\mathrm{C}=\mathrm{A} \mathrm{t}^{\mathrm{n}}$ (ecuación (2), descrita en el apartado de Introducción), con unos exponentes n que prácticamente todos ellos se encuentran en el intervalo 0,8-1,0. Para poder estimar la velocidad de corrosión a largo plazo y elaborar el mapa, se ha empleado una ecuación de este tipo, donde A (velocidad de corrosión anual) se obtiene del apartado 3.1, mientras que los valores de $\mathrm{t}$ y $\mathrm{n}$ se obtienen realizando una media de los datos obtenidos y recopilados en el estudio citado anteriormente ${ }^{[6]}$. De esta manera, tomados un valor medio de $t=15$ años, y un valor medio de $\mathrm{n}=0,9$, la ecuación empleada ha sido;

$$
C=A \cdot 15^{0,9}
$$

donde $\mathrm{C}$ es la corrosión a largo plazo, en $\mu \mathrm{m}$, y A la corrosión en $\mu \mathrm{m}$ durante el primer año de exposición.

El mapa obtenido se muestra en la figura 4. De acuerdo con la tabla III, donde se muestran los

Tabla III. Intervalos de corrosión a largo plazo (15 años) del zinc para las diferentes categorías de corrosividad ${ }^{[7]}$

Table III. Long-term (15 year) zinc corrosion intervals for different corrosivity categories ${ }^{[7]}$

\section{Categoría de Velocidad de corrosión corrosividad a largo plazo (15 años) del zinc, $\mu \mathrm{m}$}

\begin{tabular}{lc} 
C1 (muy baja) & $\mathrm{v}_{\mathrm{Zn}} \leq 1,25$ \\
C2 (baja) & $1,25<\mathrm{v}_{\mathrm{Zn}} \leq 7,5$ \\
C3 (media) & $7,5<\mathrm{v}_{\mathrm{Zn}} \leq 30$ \\
C4 (alta) & $30<\mathrm{v}_{\mathrm{Zn}} \leq 60$ \\
C5 (muy alta) & $60<\mathrm{v}_{\mathrm{Zn}} \leq 150$ \\
\hline
\end{tabular}

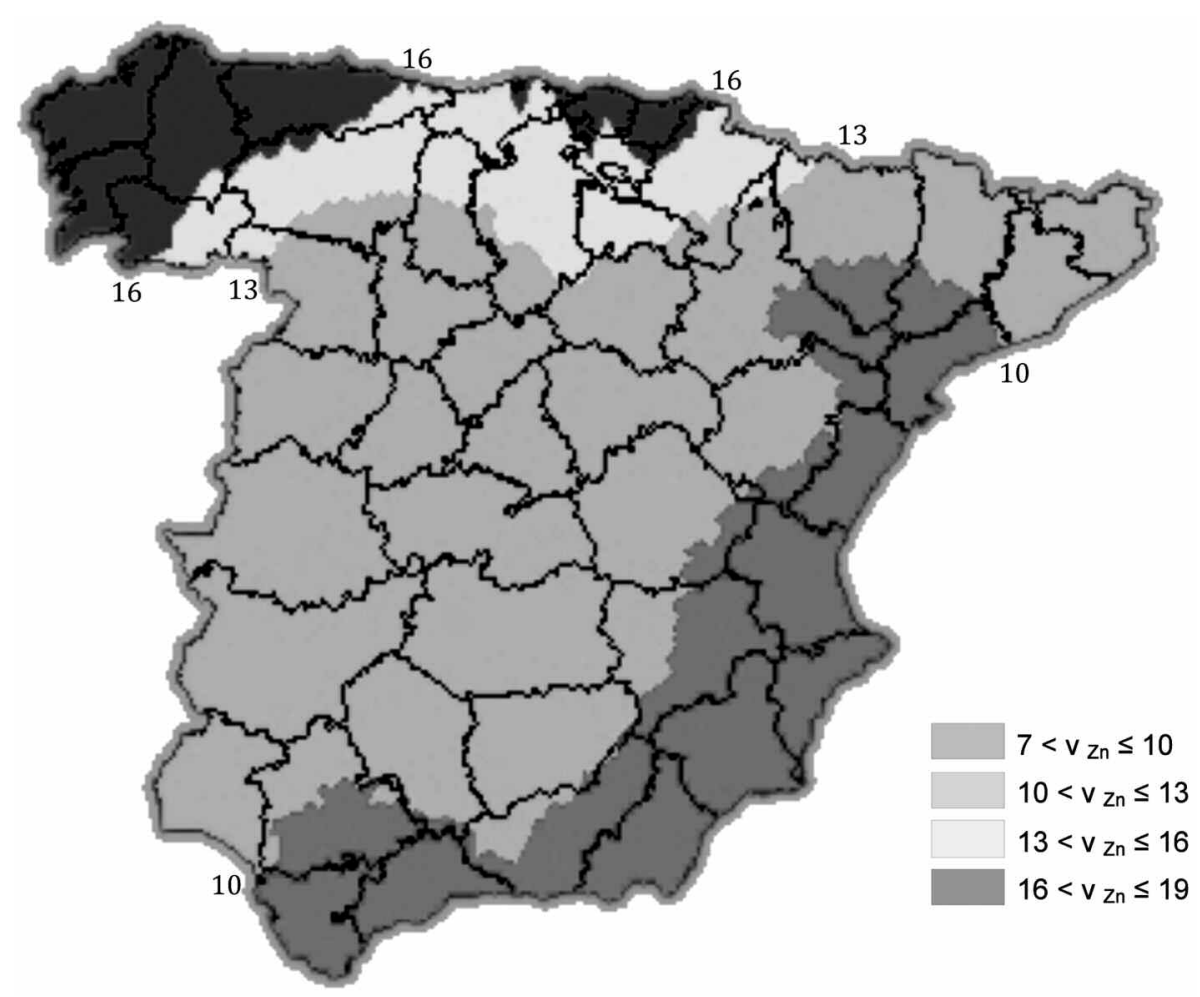

Figura 4. Mapa de España de velocidad de corrosión a largo plazo (15 años) del zinc $\left(V_{Z n}\right.$ en $\left.\mu \mathrm{m}\right)$.

Figure 4. Map of Spain for long-term (15 year) zinc corrosion rate $\left(V_{Z n}\right.$ in $\mu \mathrm{m})$. 
distintos intervalos de velocidad de corrosión a largo plazo del zinc que establece la norma ISO 9224[7] para las distintas categorías de corrosividad y 15 años de exposición, las velocidades de corrosión obtenidas se corresponderían prácticamente en su totalidad con la categoría C3. Este intervalo de velocidad establecido por ISO (concretamente entre el límite inferior y medio), se ha dividido a su vez en cuatro intervalos, con un máximo de velocidad de corrosión obtenido de 18,4 $\mu \mathrm{m}$ : (a) $7<\mathrm{v}_{\mathrm{Zn}}$ $\leq 10 \mu \mathrm{m}$; (b) $10<\mathrm{v}_{\mathrm{Zn}} \leq 13 \mu \mathrm{m}$, (c) $13<\mathrm{v}_{\mathrm{Zn}} \leq 16$ $\mu \mathrm{m}$ y (d) $16<\mathrm{v}_{\mathrm{Zn}} \leq 19 \mu \mathrm{m}$ para las distintas regiones de España.

\section{CONCLUSIONES}

De acuerdo con los mapas obtenidos para España de velocidad de corrosión anual del zinc (atmósferas rurales), se puede concluir que, a excepción de las provincias de Murcia y Almería, donde se obtiene una categoría de corrosividad C2 (baja), de acuerdo a ISO 9223, para el resto de las atmósferas rurales de España se obtiene una categoría de corrosividad C3 (media). En este intervalo de velocidad que establece la norma ISO $\left(0,7<v_{z_{n}} \leq 2,1\right.$ $\mu \mathrm{m} / \mathrm{año}$ ), se han considerado a su vez tres intervalos: (a) $0,7<v_{Z n} \leq 1,0 \mu \mathrm{m} /$ año; (b) $1,0<v_{Z n} \leq 1,3$ $\mu \mathrm{m} / \mathrm{año}$, (c) $1,3<\mathrm{v}_{\mathrm{Zn}} \leq 1,7 \mu \mathrm{m} /$ año para las distintas regiones de España.

\section{Agradecimientos}

Los autores quieren agradecer a BP Solar España, SAU, la financiación del presente trabajo.

\section{REFERENCIAS}

[1] S. Feliu y M. Morcillo, Corrosión y protección de los metales en la atmósfera, Ed. Bellaterra, Barcelona, España, 1982.

[2] M. Morcillo y S. Feliu, en Mapas de España de Corrosividad Atmosférica, M. Morcillo y S. Feliu (Eds.), Programa CYTED, Madrid, España, 1993, pp. 57-99.

[3] S. Feliu y M. Morcillo, Corros. Sci. 34 (1993) 403-414.

[4] S. Feliu, M. Morcillo y S. Feliu Jr., Corros. Sci. 34 (1993) 415-422.

[5] ISO 9223: Corrosion of metals and alloys. Corrosivity of atmospheres. Classification, ISO, 1992.

[6] M. Morcillo, J. Simancas y S. Feliu, Atmospheric Corrosion, W.W Kirk y H.H. Lawson (Eds.), ASTM STP 1239, Philadelphia, EEUU, 1995, p. 195.

[7] ISO 9224: Corrosion of metals and alloys. Corrosivity of atmospheres. Guiding values for the corrosivity categories, ISO, 1992.

[8] L. Mariaca y M. Morcillo, en Corrosión y protección de metales en las atmósferas de Iberoamérica. Parte I, M. Morcillo, E. Almeida, B. Rosales, J. Uruchurtu y M. Marrocos (Eds.), Programa CYTED, Madrid, España, 1998, pp. 629-660.

[9] "Atlas Nacional de España. 2ª Edición. Climatología”, Instituto Geográfico Nacional, Ed. Centro Nacional de Información Geográfica, 2005.

[10] J.G. Castaño, C.A. Botero y S. Peñaranda, Rev. Metal. Madrid 43 (2007) 133-145.

[11] D. de la Fuente, J.G. Castaño y M. Morcillo, Corros. Sci. 49 (2007) 1.420-1.436. 